JOURNAL DE PHYSIQUE

Colloque C2, supplément au $n^{\circ} 2$, Tome 46 , février 1985

page C2-647

\title{
DETERMINATION OF PROTON BEAM POLARIZATION AT HIGH ENERGIES BY MEASUREMENTS AFTER DECELERATION
}

J. Bystricky, F. Lehar, A. de Lesquen, A. Penzot, L. Van Rossum,

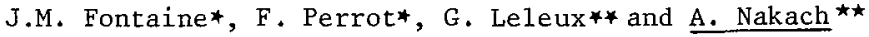

DPhPE, CEN-Saclay, France

* DPhP/ME, CEN-Saclay, France

** Laboratoire National Saturne, CEN-Saclay, France

Istituto Nazionale di Fisica Nucieare, Trieste, Italy

Résumé Nous étudions à SATURNE II une nouvelle méthode pour déterminer la polarisation d'un faisceal de protons à haute énergie après décélération à une énergie plus basse où 11 est possible a'utiliser des techniques simples et précises qui jont appel au pouvoir analyseur important et bien connu de la diffusion élastique proton-proton. Cette méthode exige de bien connaitre la dépolarisation dụ faisceau pendant la décélération. Une première mesure a montré que la dépolarisation lors du passage de la résonance $\gamma G=3$ à $631 \mathrm{MeV}$ en décélération était la même qu'en accélération. Une deuxième mesure a montré que l'égalité des dépolarisations en montée et en descente était réalisée avec une bonne approximation également lors du passage successif des deux résonances $\gamma \mathrm{G}=\nu_{\mathrm{z}} \mathrm{a} \simeq 900 \mathrm{MeV}$ et $\gamma \mathrm{G}=4$ à $1145 \mathrm{MeV}$.

Abstract we are investigating a new method to determine the polarization of proton beams accelerated to high energies by measurements after deceleration to low energies where simple and precise techniques can be used based on the large and well known analyzing power of pp elastic scattering. The polarizeo proton beam of SATURNE II was accelerated to $520 \mathrm{MeV}$ and its polarization was measured by extracting the beam onto the $\mathrm{N}-\mathrm{N}$ beam line polarimeter. The beam was then acceierated to $800 \mathrm{MeV}$, decelerated to $520 \mathrm{MeV}$ and again extracted. The loss in polarization is due to crossing twice the intrinsic depolarizing resonance $\gamma \mathrm{G}=3$ at $631 \mathrm{MeV}$ with adiabatic spin flip, once during acceleration and once during deceleration. The depolarization was intentionnaly increased by partially correcting the resonance, thus making the adiabatic flip less complete. The correction was introduced either at the rise or at the descent. The final polarization was the same in both cases showing that the depolarization, as expected, was the same during acceleration and deceleration. Another measurement was performed between 880 and $1200 \mathrm{MeV}$ crossing successively two intrinsic resonances $\gamma \mathrm{G}=v_{z}$ at $\simeq 900 \mathrm{MeV}$ and $\gamma \mathrm{G}=4$ at $1145 \mathrm{MeV}$. Here the polarization at $1200 \mathrm{MeV}$ was measured directly and is compared to the value calculated from the measurements at $880 \mathrm{MeV}$ before accelerating to $1200 \mathrm{MeV}$ and after decelerating from $1200 \mathrm{MeV}$, assuming symmetric depolarization. The measured and the calculated values agree within $\Delta \mathrm{P}_{\mathrm{B}}=0.03$ at $\mathrm{P}_{\mathrm{B}} \simeq 0.75$.

\section{I - INTRODUCTION}

Deceleration of the unpolarized beam had been used at SATURNE II. Th' Figure 1 shows the absence of beam loss during acceleration and deceleration and the reproductibility of the beam phase space when returning to the same energy. This suggested that also the depolarizing mechanisms encountered during acceleration shoula reproduce during deceleration. The theory of depolarizing resonances shows in fact that the depolarization depends on the rate of change $\mathrm{dB} / \mathrm{dt}$ of the magnetic field $\mathrm{B}$ but not on the sign of $\mathrm{dB} / \mathrm{dt}$. This permits to envisage a method to determine the beam polarization at a high energy $\mathrm{E}$ by two polarization measurements at a low energy $E_{0}$, once before accelerating up to $E_{1}$ and once after returning from $\mathrm{E}_{1}$ to $\mathrm{E}_{0}$ by deceleration.

The interest of this method for experiments at SATURNE II resides in the fact that the standard technique for absolute measurement of beam polarization by elastic pp scattering in a polarimeter begins to loose its precision at energies above 800 Mev. The polarimeter [1] 


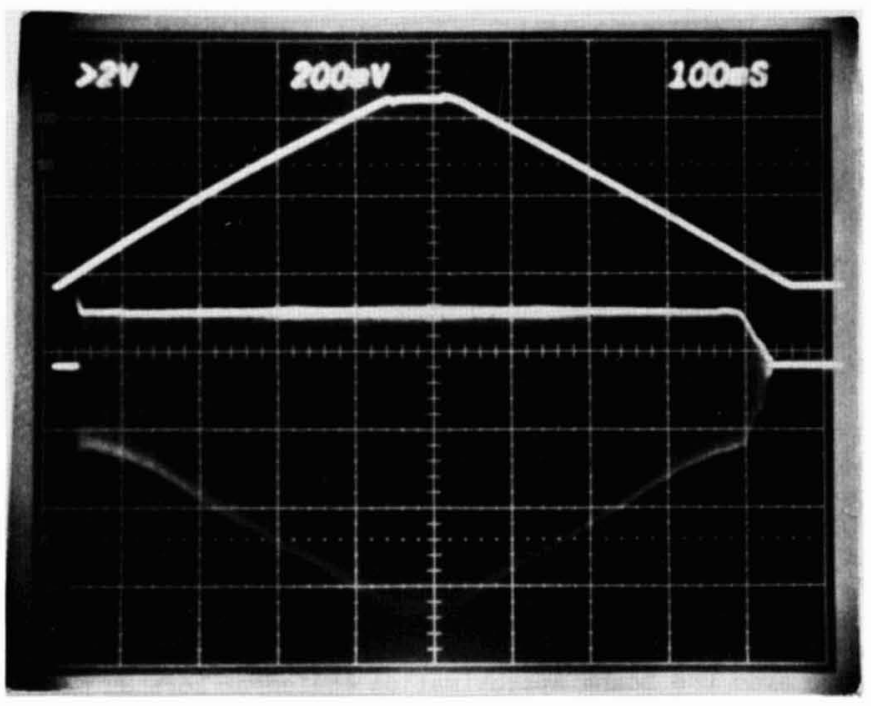

Fig.1 Acceleration of $2 \times 10^{11}$ protons to $>2 \mathrm{GeV}$ followed by deceleration. Upper trace: magnetic cycle $450 \mathrm{msec}$ rise, $100 \mathrm{msec}$ flat-top, $450 \mathrm{msec}$ descent. Lower trace: pick-up electrodes. The baseline shows the intensity, the envelop of the RF peaks shows the decrease of bunch length and the symmetric increase during deceleration.

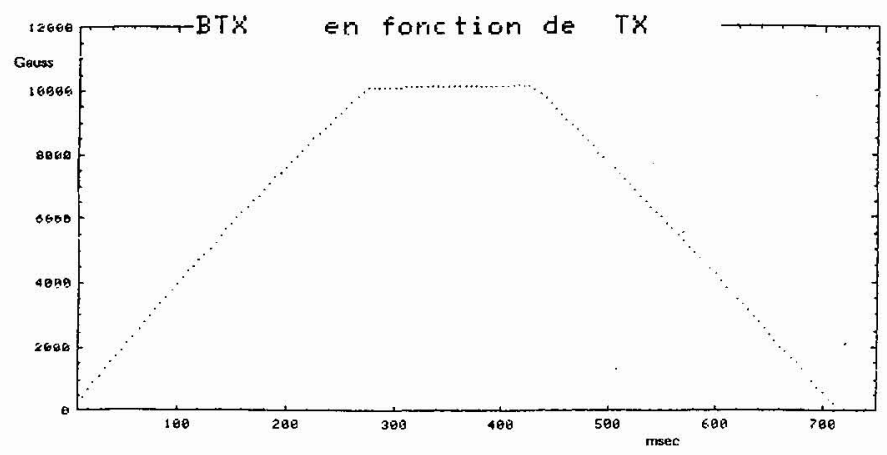

Fig.2 On-line measurements of the magnetic field. Several cycles are superposed. The dispersion is less than the size of the points. 
measures the left-right asymmetry $\epsilon_{\mathrm{pp}}$ of the elastic cross section in the plane perpendicular to the beam polarization at a center of mass angle $\Theta^{*}$ where the analyzing power $A$ is largest. The beam polarization $P_{B}$ is given by the product of asymmetry and analyzing power $\mathrm{P}_{\mathrm{B}}=\epsilon_{\mathrm{pp}}{ }^{\mathrm{A}} \mathrm{o} 0 \mathrm{no}$. Up to $800 \mathrm{MeV}$ the large number of data on pp elastic scattering yield an unique solution for an energy dependent phase shift analysis [2] from which $A_{00 n o}\left(E, \theta^{*}\right)$ is known with a precision of $\pm 3 \times 10^{-2}$ and which describes very well the direct measurements of $A_{\text {ono }}$ entering the analysis. In the region from $800 \mathrm{MeV}$ to $1 \mathrm{GeV}$ the analyzing power is still known with a precision better than $10^{-2}$, but above $1 \mathrm{GeV}$ where no phase shift analysis is presently possible, the sparse direct measurements of A $A_{0}$ with polarized proton targets have statistical errors of (5 to 10$) \times 10^{-2}$ and systematic uncertainties which may be as large as ( 10 to 15$) \times 10^{-2}$.

In principle it is possible to determine the beam polarization at SATURNE II in the region from 1 to $3 \mathrm{GeV}$ with precision of the order of $10^{-2}$ by comparing the asymmetry with polarized beam to the asymmetry with polarized target after absolute calibration of the target polarization at an energy below $800 \mathrm{MeV}$. This, however, would require a long experiment at each beam energy, with off-line analysis of the data recorded in the two-arm MwPC spectrometer with magnetic analysis detecting scatterings from the polarized target. The method of deceleration would be considerably faster and more convenient, taking advantage of the higher analyzing power at low energies and of the simplicity and on-line response of the polarimeter.

At higher energies this method may become even more interesting since the factor of merit of elastic Pp scattering, the analyzing power squared times the differential cross section, continues to decrease at all scattering angles. One estimates that methods others than pp elastic scattering are needed to measure beam polarizations above 50 or $100 \mathrm{GeV}$.

\section{II - EXPERIMENTAL CONDITIONS AT SATURNE II}

Deceleration without extraction had been used previously (Figure 1). The magnet power supply does not allow flat-top during descent and the beam had to be extracted on a short spill. The pulse-to-pulse reproductibility of the magnetic cycle is shown in Figure 2 where the on-line measurements for several cycles are superposed. These measurements provide the local slope $\mathrm{dB} / \mathrm{dt}$ at any point of the cycle. The Figure 3 shows the depolarizing resonances of sATURNE II. Closed orbit imperfection resonances $\gamma G=n(n=3, \ldots, 7)$ occur at fixed energies. The precise energy of the systematic quadrupole resonances depend on the vertical wave number $\nu_{\mathbf{z}}$ and can be changed within certain limits.

To study passing of resonances during acceleration and the symmetric process during deceleration we have chosen two energy regions: Between 520 and 800 MeV where we cross the uncorrected resonance $\gamma \mathrm{G}=3$, and between 880 and $1200 \mathrm{MeV}$ where we cross both $\gamma \mathrm{G}=\nu_{\mathrm{z}}$ and $\gamma \mathrm{G}$ $=4$.

During descent we use dynamic resonant extraction with the horizontal wave number $\nu_{\mathrm{x}}$ tuned to the desired energy. The corresponding values of the F- and D-quadrupole currefits are registred and displayed. The external beam channel is set at the nominal energy and the exact beam energy is obtained by fine-tuning of $v$, until the beam is on the center Iine up to the polarimeter. The extraction hexapoles must satisfy the condition $x=d \nu /(d p / p)=0$ in order to bring the entire beam to resonance. The Figures $4 \mathrm{a}$ and $4 \mathrm{~b}$ show the spili during descent as seen by the extracted beam monitor and by the polarimeter, respectively. The Figure $4 \mathrm{~b}$ shows the superposition of six consecutive_fursts. The leading edge jitter is of the order of 200 $\mu \mathrm{sec}$ corresponding to $\Delta \mathrm{B} / \mathrm{B}=\Delta \mathrm{p} / \mathrm{p} \approx 10^{-3}$. Pulse-to-pulse intensity fluctuations are the same as for flat-top extraction. The only serious inconvenient is the short spill which obliges us to reduce the number of protons per burst. Otherwise the operating conditions of the polarimeter such as background, empty target effect etc. are the same as on flat-top. 


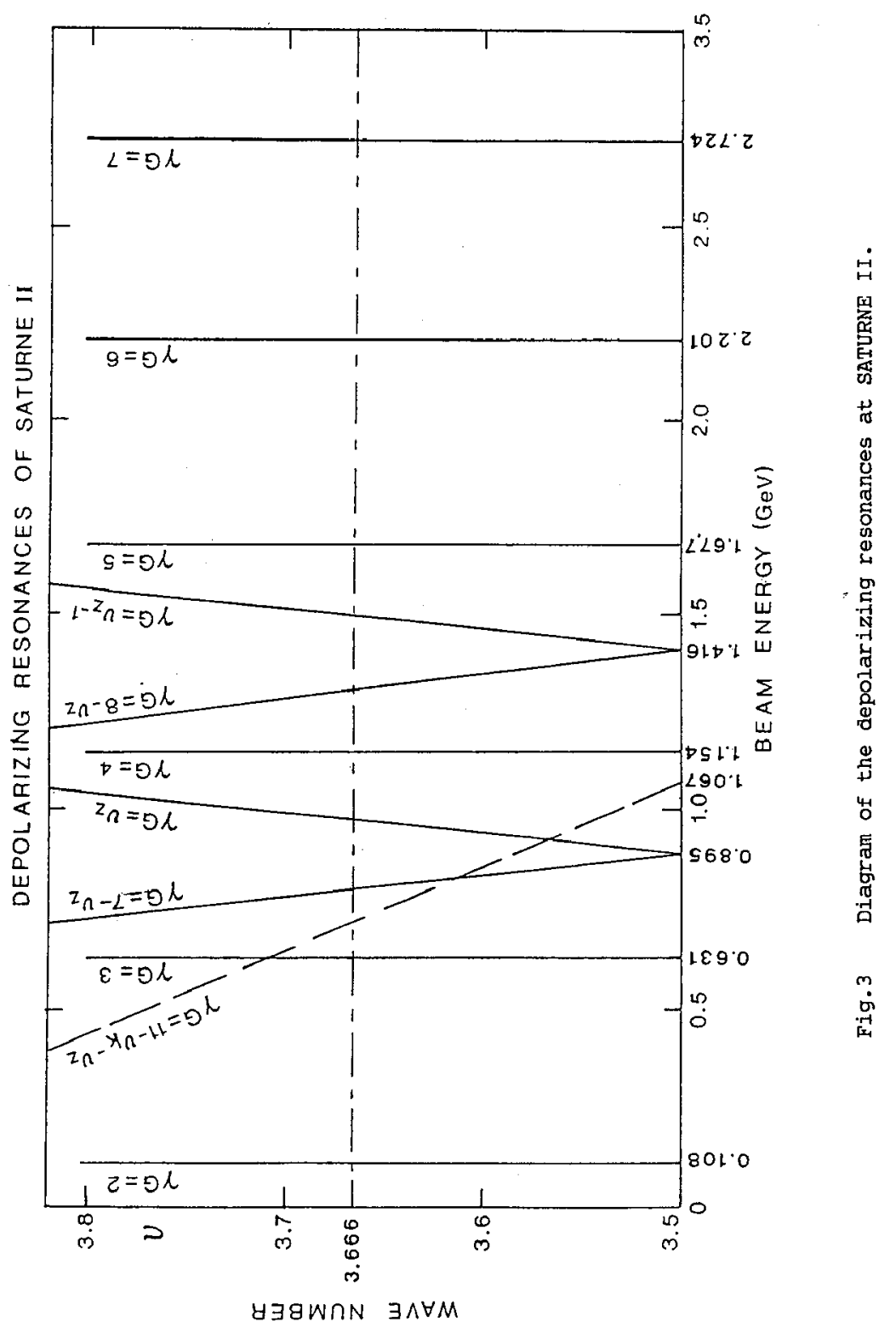




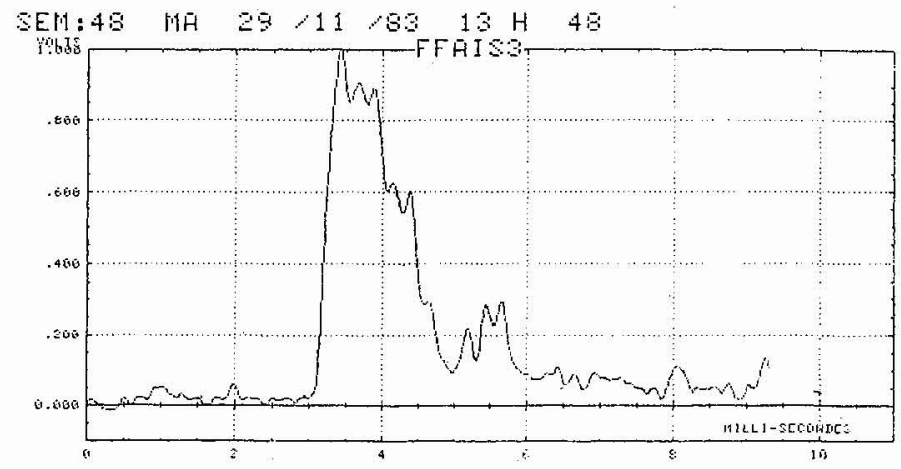

Figure 4a

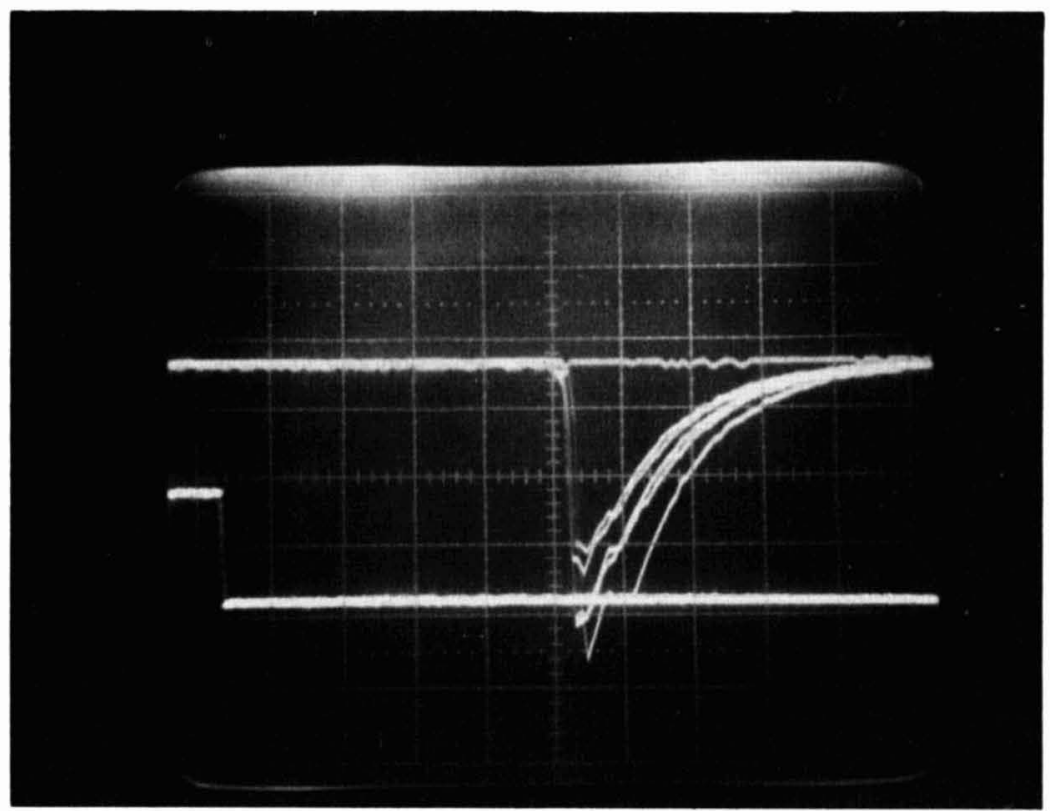

Figure $4 b$

Fig.4 Short spill at dynamic extraction during descent.

(a) External beam monitor and (b) incegrated counter signal from the polarimeter. Six bursts are superposed. Scope sweep $2.5 \mathrm{msec} /$ division. 


\section{III - RESULTS}

\section{Depolarization between 520 and $800 \mathrm{MeV}(\gamma \mathrm{G}=3$ at $631 \mathrm{MeV}$ )}

The beam was extracted on flat-top at $520 \mathrm{MeV}$ onto the polarimeter [1] in the Nucleon-Nucleon beam line. The slow spill of $200 \mathrm{msec}$ duration allowed to use (2 to 5$) \times 10^{\circ}$ particles pęr burst. The accidental rates measured between conjugate arms of the polarimeter were $\leq 2 \times 10^{-3}$. Under these conditions the results corrected for accidertal coincidences are independent of beam rate. The measured beam polarization was $\left|P_{1}\right|=0.8665 \pm 0.0043$.

The accelerator cycle was then modified for a $50 \mathrm{msec}$ flat-top at $800 \mathrm{MeV}$ without extraction, followed by deceleration and dynamic extraction at $520 \mathrm{MeV}$ with a spill of $2 \mathrm{msec}$ (Figure 4 ). The intensity was reduced by detuning the RF capture after injection to achieve an accidental rate of $\leq 3 \times 10^{-2}$ between two counters in the same polarimeter arm. This corresponds to the upper limit of instantaneous beam rate compatible with reliable measurements. The beam polarization was found to be $\left|P_{2}\right|=0.8317 \pm 0.0015$. The difference $\left|P_{1}\right|-\left|P_{2}\right|$ represents the combined loss due to crossing twice the uncorrected intrinsic depotarizing resonance $\gamma G=3$ at $631 \mathrm{MeV}$ with adiabatic spin flip, once during acceleration and once during deceleration.

The following procedure was used to check if passing the resonance during acceleration produces the same depolarization as crossing it during deceleration: First, the resonance was partially corrected both at the rise and at the descent. Reducing the strength of the resonance reduces the amount of adiabatic flip, thus increases the depolarization. By progressively increasing the correction, the polarization was found to decrease from $|P|=$ 0.83 to $|P|=0.77$. Then, the same fixed correction of the resonance was introduced either only at the rise $(R)$ or only at the descent $(D)$. The mesured beam polarizations where $|P|_{R}=$ $0.818 \pm 0.014$ and $|\mathrm{P}|_{\mathrm{D}}=0.819 \pm 0.019$, respectively. This result is consistent with perfectly symmetric behaviour of the beam polarization when passing the same resonance during acceleration or deceleration. The conclusion is independent of $A$ ons since all measurements are performed at the same energy and center of mass angle. The relatively large statistical errors in these two measurements are due to the fact that the SATURNE magnet power supply does not allow a flat-top during descent.

\section{Depolarization between 880 and $1200 \mathrm{MeV}\left(\gamma \mathrm{G}=\nu_{z}\right.$ at $\simeq 900 \mathrm{MeV}$ and $\gamma \mathrm{G}=4$ at $\left.1145 \mathrm{MeV}\right)$}

This measurement studied the depolarization when crossing two consecutive intrinsic depolarizing resonances $\gamma G=v_{\text {at }}$ at about $900 \mathrm{MeV}$ and $\gamma \mathrm{G}=4$ at $1145 \mathrm{MeV}$. The beam polarization (Table 1 and Figure 5) was measured by slow extraction on flat-top at (a) $880 \mathrm{MeV}$ and (b) 1200 $\mathrm{MeV}$, and (c) by dynamic extraction at $880 \mathrm{MeV}$ during dèceleration from $1200 \mathrm{MeV}$. The initial polarization at $880 \mathrm{MeV}$ was $\left|\mathrm{P}_{2}\right|=0.8938$, the polarization at $1200 \mathrm{MeV}$ was $\left|\mathrm{P}_{b}\right|=0.7277 \mathrm{MeV}$ and the polarization at $880 \mathrm{MeV} \mathrm{P}^{2}$ after deceleration was $\left|P_{C}\right|=0.6798$.

The absolute value of the rate of change of the magnetic field was not exactly the same during rise and descent. The measured values are $(\mathrm{dB} / \mathrm{dt})_{R}=+3.013 \mathrm{Tesla} \mathrm{x} \mathrm{sec}^{-1}$ and $(\mathrm{dB} / \mathrm{dt})_{\mathrm{D}}=-2.50$ Tesla $\mathrm{x} \mathrm{sec}{ }^{-1}$. A simple interpolation between the two low energy measurements (a) and (c) taking into account this difference in slope but assuming otherwise exactly symmetric depolarization predicts a polarization $\left|P_{p}\right|$ (calc.) $=0.760 \pm 0.005$. The calculated value and the direct measurement agree within $\Delta \mathrm{P}=8.032$, i.e. within 4 percent.

At the upper end of the SATURNE energy range a determination of beam polarization with an relative error of 4 percent would already be a substantial improvement over the direct measurement affected by larger errors due to the uncertainty on $A_{\text {ono }}$ at nigh energies.

For the present measurement we have estimated an upper limit for the ratio of the analyzing powers at 880 and $1200 \mathrm{MeV}$, respectively, with the conclusion that the difference $\Delta \mathrm{P}=0.032$ is probably significant. This would mean that depolarization symmetry is a useful approximation but that it was not exactly realized in this experiment.

Although the evidence for asymmetry is very weak in this measurement we further discuss the point in view of its interest for detailed understanding of depolarizing mechanisms. During the measurements we made the following observation: For the initial measurement at $880 \mathrm{MeV}$ 


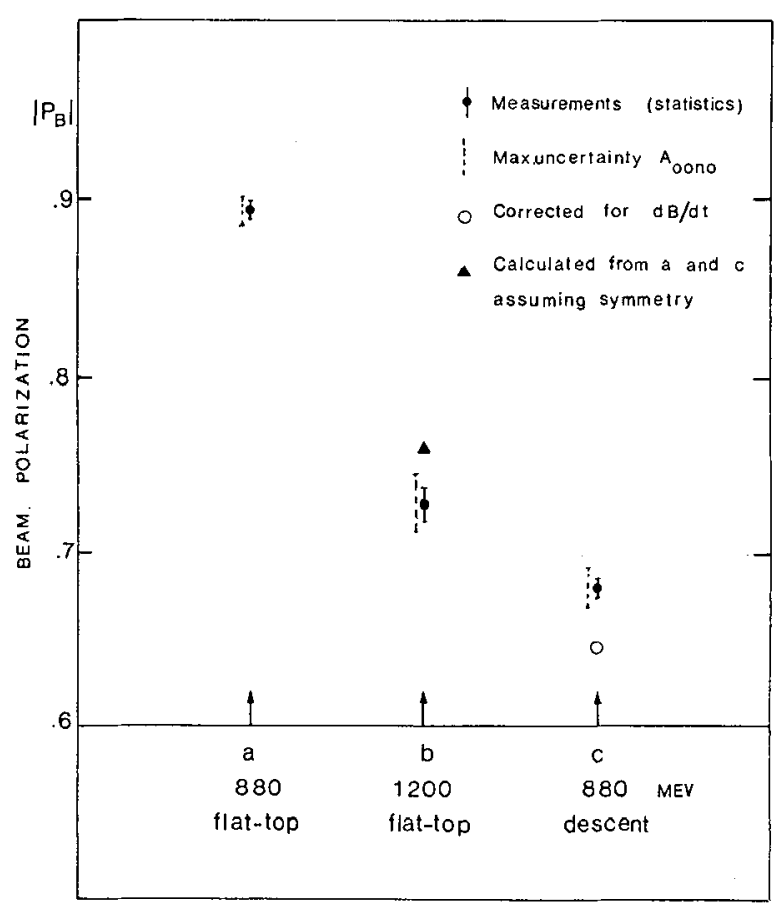

Fig.5 Depolarization between 880 and $1200 \mathrm{Mev}$. Comparison of the direct measurement at $1200 \mathrm{MeV}$ with the value calculated from the measurements at $880 \mathrm{Mev}$ before acceleration and after deceleration.

\begin{tabular}{|c|c|c|c|c|c|}
\hline & $\begin{array}{c}\text { Energy } \\
\text { MeV }\end{array}$ & Extraction & \multicolumn{3}{|c|}{$\begin{array}{c}\text { Beam Polarization } \\
\text { Statist. }\end{array}$} \\
\hline$\left|P_{a}\right|$ & 880 & Flat-top & 0.89385 & \pm 0.00296 & \pm 0.0055 \\
$\left|P_{b}\right|$ & 1200 & Flat-top & 0.72772 & \pm 0.00866 & \pm 0.0180 \\
$\left|P_{c}\right|$ & 880 & Descent & 0.67982 & \pm 0.00417 & \pm 0.0103 \\
\hline$\left|P_{d}\right|^{*}$ & 1200 & Flat-top & 0.58045 & \pm 0.00916 & \pm 0.0144 \\
\hline
\end{tabular}

* With cut-off in vertical betatron oscillation amplitude.

Table 1 Beam polarization measurements at 880 and $1200 \mathrm{MeV}$. The systematic errors correspond to upper limits for uncertainties of the analyzing power used to calculate the polarizations from the measured asymmetries. 
the pulse-to-pulse intensity fluctuations had been stabilized by a standara prccedure which at this energy had no effect on beam polarization. When changing to 1200 MeV we first found an unusually low polarization of only $|P|=0.5805$. We discovered that removing the stabilizer increased the polarization to $|\mathrm{P}|=0.7277$. The procedure limits the intensity fluctuations by a cut-off in the vertical betatron oscillations shortly after injection. No change in polarization was observed when producing the same reduction in intensity by an alternate method, detuning the RF capture. A systematic study of this effect was subsequently carried out [3] by accelerating through two successive quadrupole resonances $\gamma G=\nu$ and $\gamma G=8-v$ with precise polarization measurements on flat-top only. The results are suggesting the following mechanism. A cut-off on the vertical betatron oscillations leaves the core unchanged but reduces the peripheral part. On the other hano, when crossing the quadrupole resonance with adiabatic flip it is reasonnable to expect that only the peripheral part experiences a strong resonance wile the core sees a very weak resonance and does not flip. The intermediate phase space sees a resonance too weak for adiabatic flip but strong enough to depolarize. The observed depolarization when passing an odd number of quadrupole resonances with adiabatic flip is actually composed of a true loss corresponding to statistical disorder and an apparent loss since different phase space regions have opposite polarizations. The polarimeter in the extracted beam measures the average polarization of all particles.

In our case, after crossing the resonances again during deceleration, the losses due to $\gamma \mathrm{G}=$ 4 and the true losses due to $\gamma \mathrm{G}=\nu_{\mathrm{z}}$ cumulate, but not the apparent losses since the second adiabatic flip restores the same sign for core and halo. The expected depolarization asymmetry is very small since we worked at full betatron amplitude. The effect becomes appreciable only when severely limiting the vertical phase space [3].

\section{IV - GONCLUSIONS}

Determination of the polarization of proton beams accelerated to high energies by measurements at lower energies before acceleration and after deceleration requires detailed study of resonant depolarization. We have used well known resonances of SATURNE II as test cases. The study concerns strong resonances inducing adiabatic spin flip. The depolarizations are the same during acceleration and deceleration for the closed orbit imperfection resonances $\gamma G=n(n=3, \ldots, 7)$. For the systematic quadrupole resonances we observe almost symmetric behaviour. However, this case especially requires additional studies and computer simulation to achieve the understanding necessary for precise calculation of the polarization at high energy from the measurements at low energy. The present results confirm that this is a very promising method which becomes very simple and fast in the case of an accelerator capable of flat-top on descent. Other methods for direct beam polarization measurements at nigh energy are being developped. We feel that reducing the problem to an already solved problem at low energy gives the method of deceleration an obvious competitive advantage.

\section{ACKNOWLEDGEMENTS}

The authors wish to thank Professor M.Jean for his active interest and encouragement.

\section{REFERENCES}

[1] M.Arignon et al, Note CEA-N-2375, Saclay, December 1983

[2] J.Bystricky, C.Lechanoine-Leluc and F.Lehar, Saclay Report DPhPE 82-12, December 1982

J.Bystricky and F.Lehar, Nucleon-Nucleon Scattering Data, KFZ Karlsrune, Physics Data, Vols. 1,2 and $3(1978,1981)$ H.Behrens and G.Ebel, editors

J.Bystricky et al., Landolt-Bornstein Tables, New Series Vol.9 (1980), editor H.Schopper, Springer Verlag

[3] A.Nakach, Influence of Betatron Oscillations on the Protor Beam Polarization, contributed paper, The 6 th International Symposium on High Energy Spin Physics, Marseille, 12-19 Sept.84 